\title{
CHARACTERIZATION OF 2D AND 3D ROUGH FRACTAL SURFACES FROM BACKSCATTERED RADAR DATA
}

\author{
Apostolos KOTOPOULIS, Georgios POURAIMIS, \\ Evangelos KALLITSIS, Panayiotis FRANGOS
}

\author{
National Technical University of Athens, Athens, Greece \\ pfrangos@central.ntua.gr
}

\begin{abstract}
The scattering of electromagnetic (EM) waves, emitted by a monostatic radar, from two dimensional (2D) rough fractal surfaces is examined by using the Kirchhoff approximation. We examine the way that the level of roughness of the fractal surface affects the backscattered EM wave captured by a radar as a function of frequency (therefore, a 'spectral method') and whether the roughness of the surface can be estimated from these radar measurements. The backscattering coefficient is calculated for a number of radar frequencies and for different values of the surface fractal dimension. It is found that the values of the slopes between the main lobe and the first sidelobes of the backscattering coefficient as a function of the wave number (frequency) of the incident EM waves increase with the surface fractal dimension. Therefore, we conclude that the magnitude of the above slopes provides a reliable method for the classification of the rough fractal surfaces. Furthermore, concerning three - dimensional (3D) fractal surfaces, investigations similar to the above are already performed by the authors and will be presented during the Conference. The above are also investigated in the presence of electronic noise in the radar receiver (effect of SNR values in the above proposed technique).
\end{abstract}

\section{Keywords: Radar, Scattering of electromagnetic (EM) waves}

\section{Introduction}

The scattering of electromagnetic (EM) waves from rough surfaces has been for decades a very interesting subject for scientific investigation. In many cases the main purpose of this research is the characterization of rough surfaces from scattered EM wave data for remote sensing applications, in the microwave or optical regime [1]-[14]. These surfaces can be modelled mathematically with deterministic or random functions [1]-[3]. However, introducing the fractal geometry, these surfaces can be described in a more detailed way in multiscale [1], [3], [8].

In this paper the scattering of EM waves from rough surfaces using the Kirchhoff approximation is examined [1], [2]. In particular, in Section II the mathematical fundamentals for scattering of EM waves from fractal surfaces are summarized [1]-[3]. In Section III our simulation results for the characterization of the rough fractal surfaces from backscattered EM wave data are presented. Finally, conclusions and future related research of ours are described in Section IV.

\section{Problem Geometry and Mathematical Formulation}

The geometry of the problem is shown in Fig. 1. An incident EM plane wave illuminates a one-dimensional rough fractal surface extending from $x=-L$ to $x=L$, as shown in Fig. 1.The angle of incidence of the EM wave is $\theta_{i}$ with respect to the vertical $\mathrm{z}$ axis, where the incident and scattered wave vectors are denoted by $k_{i}$ and $k_{s}$ respectively [1]. 


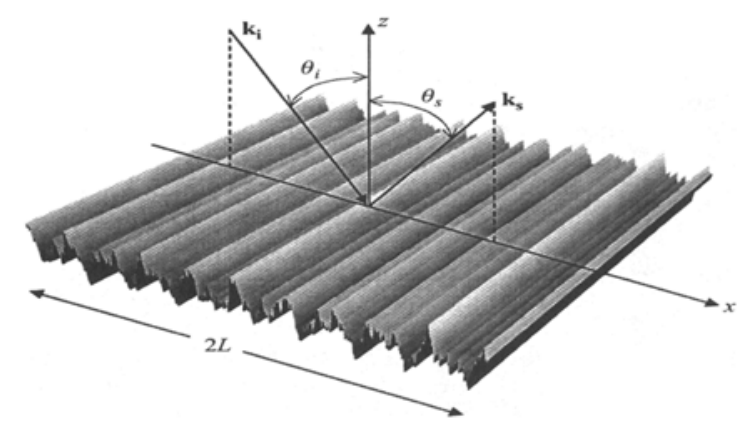

Figure 1: Geometry of the rough surface scattering problem

Following [1], and in order to describe the surface roughness, a one-dimensional fractal function is used [1], [3], [4]. This fractal function is described by the following equation:

$$
f_{r}(x)=\sigma C \sum_{n=0}^{N-1}(D-1)^{n} \sin \left(K_{0} b^{n} x+\phi_{n}\right)
$$

where $D(1<D<2)$ is the fractal dimension of fractal surface [1], $K_{0}=2 \pi / \Lambda_{0}$ is the fundamental spatial wavenumber of the fractal surface, $\Lambda_{0}$ is the corresponding fundamental spatial wavelength, $b$ (where $b>1)$ is the spatial frequency scaling parameter, ${ }_{n}$ are arbitrary phases and $N$ is the number of tones describing the surface. The amplitude control factor $C$ is given by:

$$
C=\left\{\frac{2 D(2-D)}{\left[1-(D-1)^{2 N}\right]}\right\}^{\frac{1}{2}}
$$

so that surface function (1) has standard deviation (rms height) equal to $\sigma$ [1]. It can be easily realized from (1) above, that when the surface fractal dimension $D$ increases from value 1 to value 2 , the surface roughness also increases [1], [3].

In order to calculate the scattered field from a rough fractal surface, described in Fig. 1, the Kirchhoff approximation is used, for which it is assumed that the wavelength of the incident EM wave is small compared to the local radius of curvature of the surface roughness [1]-[3]. Furthermore, for the plane EM wave incidence of Fig. 1, in [1] it is shown that the scattered electric field is given by the following equation:

$$
E_{s c}=\frac{i k L \exp \left(k R_{0}\right)}{2 \pi R_{0}} \int_{-L}^{L}\left(p f_{r}^{\prime}-q\right) \exp \left[v_{x} x+i v_{z} f_{r}(x)\right] d x
$$

$$
\begin{aligned}
& p=(1-R) \sin \theta_{i}+(1+R) \sin \theta_{s} \\
& q=(1+R) \cos \theta_{s}-(1-R) \cos \theta_{i} \\
& v_{x}=k\left(\sin \theta_{i}-\sin \theta_{s}\right) \\
& v_{z}=-k\left(\cos \theta_{i}+\cos \theta_{s}\right)
\end{aligned}
$$

In the above (3)-(7), $R_{0}$ is the distance from the observation point (monostatic radar) to the origin, coinciding with the 'source surface point', $k$ is the wavenumber of the incident EM wave $(k=2 \pi f / c$, where $f$ is the frequency of the incident EM wave), $R$ is the Fresnel reflection coefficient of the tangential plane at the point of interest, $\theta s$ is the direction of the observer and $f_{r}^{\prime}$ is the derivative with respect to its argument $x$. For simplicity we assume a perfectly conducting rough surface, in which the Fresnel reflection coefficient is given by $\left(R^{+}=1, R^{-}=1\right)$, where the superscript + indicates the parallel (vertical) polarization and the superscript - denotes the perpendicular (horizontal) polarization, respectively [1], [2].

In the case of a smooth, perfectly conducting surface, the scattered field for horizontal polarization can be found in the direction of specular reflection, namely for $\theta_{i}=\theta_{s} \quad[1],[2]:$

$$
E_{s c 0}=\frac{i 2 k L^{2} \exp \left(i k R_{0}\right) \cos \theta_{\iota}}{\pi R_{0}}
$$

Normalizing the value of the scattered field $E_{\text {sc }}$ of (3) by the value provided by (8), the scattering coefficient $\gamma$ is calculated by [1]:

$\gamma=\frac{E_{s c}}{E_{s c 0}}=\frac{1}{4 L \cos \theta_{l}} \times$

where:

$\left(\left(q+\frac{p v_{x}}{v_{z}}\right) \times \int_{-L}^{L} \exp \left[i v_{x}+i v_{z} f_{r}(x)\right] d x-\left\{\frac{\dot{p}}{v_{z}} \exp \left[i w_{x} x+i v_{z} f_{r}(x)\right]\right\}_{-L}^{L}\right)$ 
The first term in the parenthesis provides the most significant contribution to the scattering process, while the second term represents an edge effect, which can be considered negligible when $L>\lambda$, as assumed in this paper.

\section{Simulation Results}

We concentrate on the backscattering of EM waves from rough fractal surfaces (e.g. monostatic SAR radar [9], [10]), i.e. $\theta_{s}=-\theta_{i}$ at Fig. 1 and (4)-(7) and we plot the magnitude of the backscattering coefficient $|\gamma(k)|$. The surface is simulated as a zeromean, band-limited fractal function, as in (1), and its roughness is controlled by the fractal dimension $D$ [1], [3]. The backscattering coefficient $\gamma$ was calculated from (9) for a number of frequencies, $f_{m}=f_{o}+(m-1) \Delta f$, where $m=1,2, \ldots, M$ and $M$ is the number of frequencies, $f_{o}$ is the carrier frequency, $\Delta f=B W / M$ is the frequency step and BW is the bandwidth of the radar, i.e. 'stepped - frequency' transmitted radar waveform [9], [10].

\subsection{Noiseless Receiver}

In Fig. 2, below, the plots of $|\gamma(k)|$ for angle of incidence $\theta_{i}=30^{\circ}$ are shown, while the values of the other parameters are $B W=1 \mathrm{GHz}, f_{0}=10 \mathrm{GHz}$ and $M=200$ (i.e. 200 frequency steps in radar emitted stepped-frequency waveform).

As far as the simulated fractal surface is concerned, the frequency scaling parameter was set equal to $b=1.8$ while the number of tones was set equal to $N=6$ [1]. Moreover, the rms height of the surface was set equal to $\sigma=0.05 \lambda, \Lambda_{0}=10 \lambda=0.3 \mathrm{~m}$ and the illuminated length of the rough surface along $x$-direction ('patch size') was chosen to $2 L=80 \lambda$ (Fig. 1) in all calculations (so as $2 L>>\Lambda_{0}$ and $\left.k \sigma<1\right)$, where $\lambda=c / f_{0}$ [1], [18]. Furthermore, at the top left corner of each figure a sample plot of the roughness fractal function $f_{r}(x)(1)$ is also shown.

The roughness of the simulated fractal surface (the fractal dimension $D$ ) is increasing per image, e.g. $D=1.05$ (Fig. 2), $D=1.30$ (Fig. 3) and so on.

By observing Fig. 2 the following conclusion is made: as the value of the parameter $D$ increases, i.e. as the roughness of the fractal surface increases, the emerging slope between the main lobe and the side lobes also increases.

Therefore, it becomes clear in our simulations that the roughness of the fractal surface can be characterized by the mean slope between the main lobe of function $|\gamma(k)|$ and the two sidelobes, adjacent to the main lobe (see plots of Fig. 2).

If the radar bandwidth decreases, then the information provided by the backscattered signal-wavenumber plots, of the type provided above, is not always enough in order to draw safe conclusions regarding the roughness (fractal dimension) of the surface. In other words, the bandwidth for our proposed method of surface characterization from backscattered radar data must be sufficiently large (at least 5\% of the carrier frequency $f_{0}$ ), in order for the information contained in the plots of Figs. 2 to be observable and measurable. 

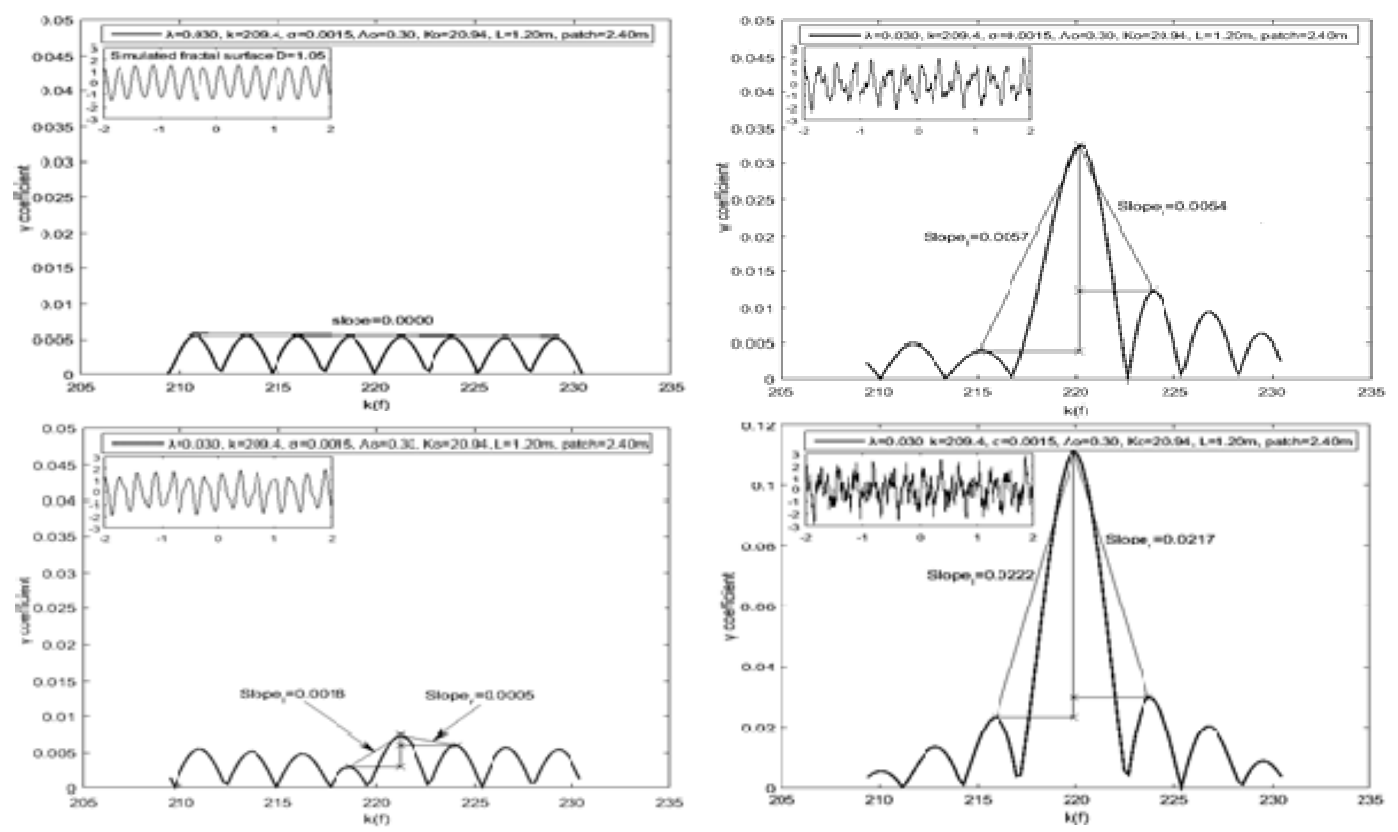

Figure 2: Magnitude of backscattering coefficient $|\gamma(k)|$, as a function of the wavenumber $k$, for $D=$ 1.05 (up left), $D=1.30$ (up right), $D=1.55$ (down left), $D=1.80$ (down right)

In order to study further the relation been performed (details of it can be found between the surface fractal dimension $D$ and the slopes of the scattering coefficient $|\gamma(k)|$, some additional simulations have in [14]). This ends up with Fig. 3 results, as it will be also explained in the Conference [14]
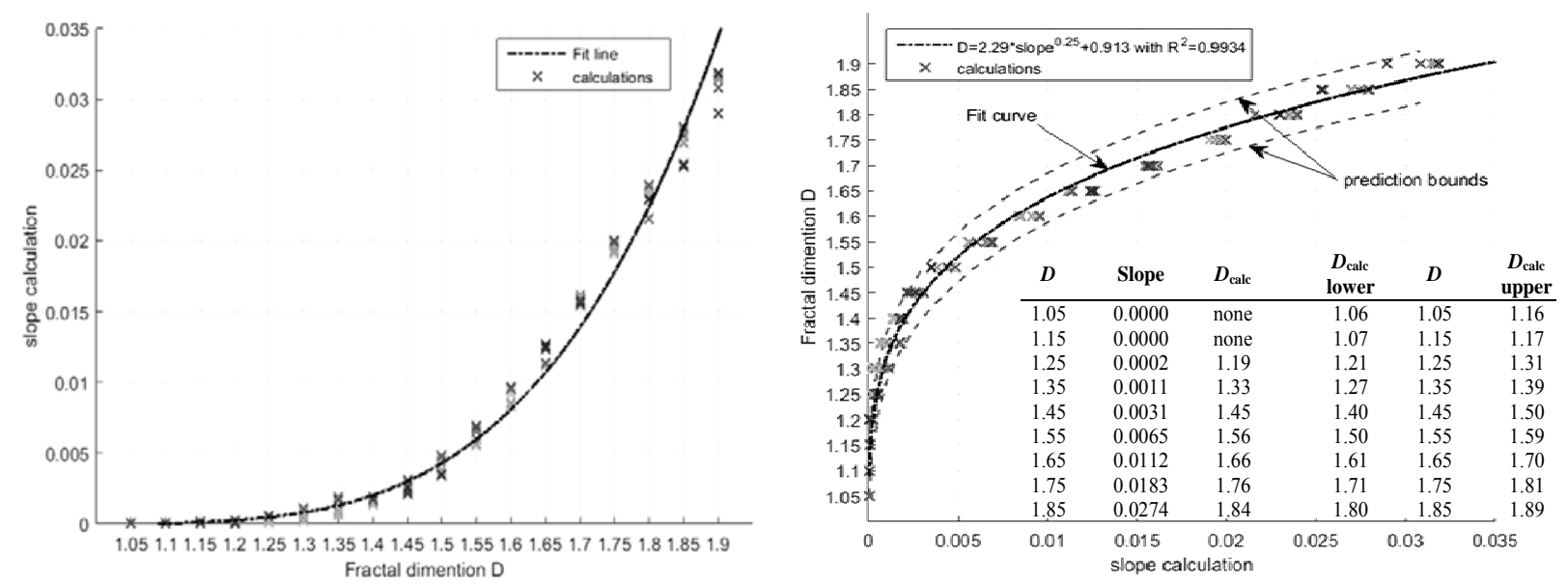

Figure 3: Left: 'Average slope' of the $|\gamma(k)|$ vs. value of the surface fractal dimension D. Right:

Fractal dimension D vs. 'slope calculation' of $|\gamma(k)|$ (simulated values and fitted curve)

\subsection{Noisy Receiver}

In order to examine the applicability of our method in an actual noisy radar environment, we add AWGN noise in $\gamma(k)$. We use (10), below, for calculating the power level $P(\gamma)$ of signal $\gamma(k)$, and (11) for calculating the noise level $N(\gamma)$ for a given Signal to Noise Ratio $(S N R)$ value.

$$
\begin{aligned}
& P(\gamma)=\frac{1}{n} \sum_{m=1}^{n}|\gamma(m)|^{2} \mid \\
& N(\gamma)=\frac{P(\gamma)}{S N R}(11) \\
& \gamma_{\text {noisy }}=\gamma+\sqrt{\frac{P(\gamma)}{S N R}} \operatorname{Norm}(0,1)
\end{aligned}
$$

The $\gamma_{\text {noisy }}$ in (12) represents a $\gamma(k)$ signal that exhibits a certain $S N R$ value using a 
Gaussian distribution with specific noise level.

From Fig. 4(a) it is obvious that a signal $\gamma(k)$ exhibiting high $S N R=23 \mathrm{db}$ does not affect our method. On the contrary a signal

(a)

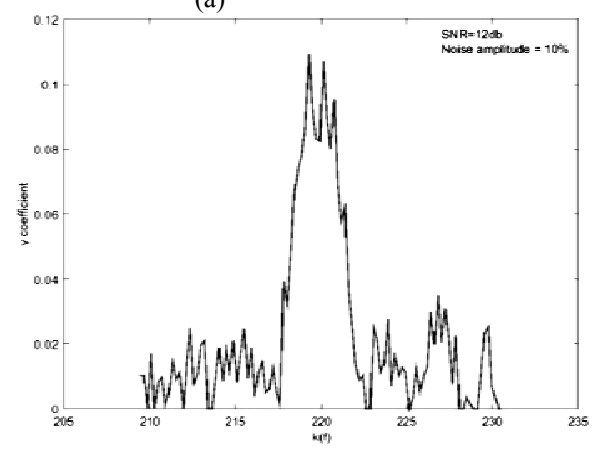

$\gamma(k)$ with low $S N R=0 \mathrm{db}$ (signal=noise), as demonstrated in Fig. 4(c), can totally suppress sidelobes and cancel our proposed method, if no additional noise suppression technique is applied.

(b)

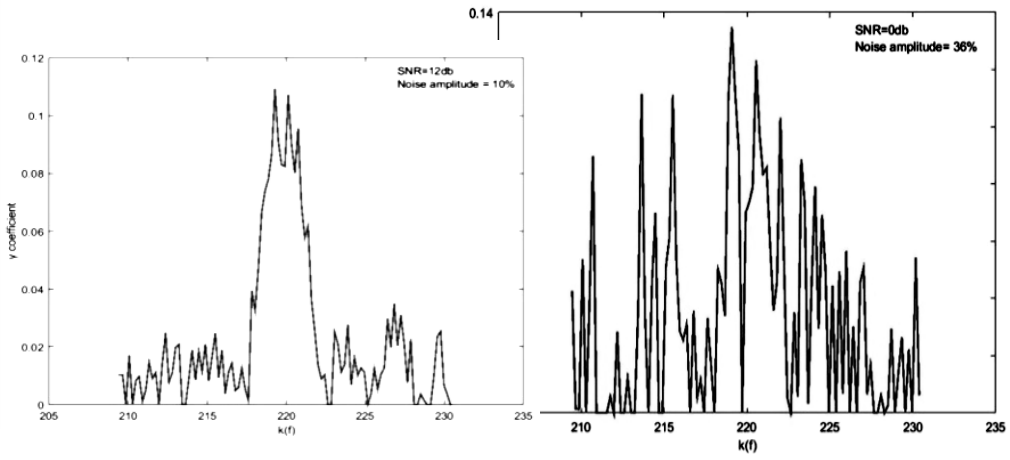

Figure 4: $\gamma(k)$ with SNR 23db inserts a noise amplitude 3\% of $|\gamma(k)|$ max amplitude; with SNR $12 d b$, inserts a noise amplitude 10\% of $|\gamma(k)|$ max amplitude and with SNR $0 d b, 36 \%$ of $|\gamma(k)|_{\max }$

There are several techniques that can be used to improve $S N R$ and make our method useful for lower $S N R$ values. Then, by averaging measurements, we were able to obtain very successful results, as reported in [15]. These will be presented in detail during the

Conference.

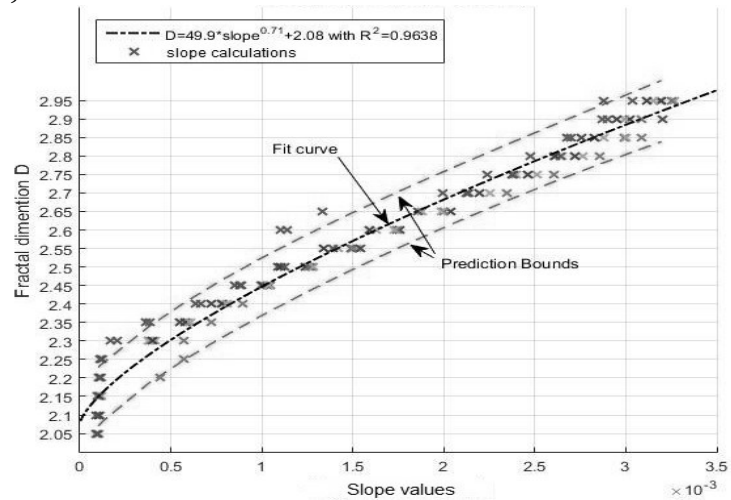

Figure 5: Value of surface fractal dimension D vs. 'slope calculation' of the $|\gamma(k)|(3 D$ case).

\section{Preliminary Results for the 3D}

Scattering Geometry

New investigations of our research group (still in progress) consider the more realistic case of 3D scattering geometry. Preliminary $3 \mathrm{D}$ fractal rough surface characterization results are shown at Fig. 5, below, and will be presented during the Conference.

\section{Conclusion}

In this paper, a novel method is presented for the characterization of rough fractal surfaces from backscattered radar data of sufficient bandwidth [9], [10]. As resulted from the plots of the backscattered signal magnitude as a function of the wavenumber (therefore a 'spectral method') of the incident EM wave, as the roughness of the fractal surface increases, then the observed slope between the main lobe and the side lobes also increases. Moreover, the fractal dimension of the surface can be estimated by the average slope of backscattering coefficient $|\gamma(k)|$. Furthermore, the value of the available radar bandwidth is crucial and must be sufficiently large, for correct rough surface characterization. Finally, we prove that our method is useful even in a noisy 
radar environment, which exhibits $\gamma(k)$ with relatively low $S N R$. It is only a matter of selecting the appropriate averaging number of bursts so as to enhance the $S N R$ of $\gamma(k)$.

\section{References}

[1] D. L. Jaggard, X. Sun, Scattering from fractally corrugated surfaces, Journal of the Optical society of America A, Vol. 7, No 6, pp. 1131-1139, 1990.

[2] P. Beckmann, A. Spizzichino, The Scattering of electromagnetic waves from rough surfaces, Artech House Inc., 1987.

[3] D. L. Jaggard, A.D. Jaggard, P. Frangos, Fractal electrodynamics: surfaces and superlattices, in Frontiers in Electromagnetics, Edited by Douglas Werner and Raj Mittra, IEEE Press, 2000, pp. 1-47.

[4] F. Berizzi, E. Dalle Mese, G. Pinelli, One dimensional fractal model of the sea surface, IEEE Proc. Radar Sonar Navig., Vol. 146, No 1, pp. 55-64, 1999.

[5] A.K. Sultan - Salem, G.L. Tyler, Validity of the Kirchhoff approximation for electromagnetic wave scattering from fractal surfaces, IEEE Trans. Geosc. Rem. Sensing, Vol. 42, No. 9, pp. 1860-1870, 2004.

[6] M. F. Chen, A. K. Fung, A numerical study of the regions of validity of the Kirchhoff and small perturbation rough surface scattering models, Radio Science, Vol. 23, pp. $163-170,1988$.

[7] E. Jakeman, Scattering by fractals, in Fractals in Physics, pp. 55-60, 1986.

[8] N. Ampilova and I. Soloviev, On digital image segmentation based on fractal and multifractal methods, CEMA'15 Conference Proceedings, pp. 14-17, Sofia, Bulgaria, 2015.

[9] A. Malamou, A. Karakasiliotis, E. Kallitsis, G. Boultadakis, P. Frangos, Application of a fully automatic autofocusing algorithm for post - processing of synthetic aperture radar images based on image entropy minimization, Electronics and Electrical Engineering Journal, Vol. 19, No. 6, pp. 95 - 98, 2013.

[10] A. Malamou, C. Pandis, P. Frangos, P. Stefaneas, A. Karakasiliotis, D. Kodokostas, Application of the modified fractal signature method for terrain classification from synthetic aperture radar images, Electronics and Electrical Engineering Journal, Vol. 20, No. 6, pp. 118-121, 2014.

[11] S. Savaidis, P. Frangos, D. L. Jaggard and K. Hizanidis, Scattering from fractally corrugated surfaces : an exact approach, Optics Letters, Vol. 20, No. 23, pp. 23572359, 1995.

[12] S. Savaidis, P. Frangos, D. L. Jaggard and K. Hizanidis, Scattering from fractally corrugated surfaces using the extended boundary condition method, Journal of the Optical Society of America A, Vol. 14, No. 2, pp. 475-485, 1997.

[13] D. L. Jaggard, X. Sun, Fractal surface scattering: a generalized Rayleigh solution, Journal of Applied Physics, Vol. 68, No 11, pp. 5456-5462, 1990.

[14] A. Kotopoulis, A. Malamou, G. Pouraimis, E. Kallitsis and P. Frangos, Characterization of rough fractal surfaces from backscattered radar data, 'Electronics and Electrical Engineering' Journal, accepted April 2016.

[15] A. Kotopoulis, G. Pouraimis, A. Malamou, E. Kallitsis, and P. Frangos, 'Characterization of fractal rough surfaces from backscattered radar data', CEMA'16, Conference, Athens, Greece, 13-15/10/2016. 